\title{
The impact of microscopic magnetic reconnection on pre-flare energy storage
}

\author{
P. A. Cassak
}

J. F. Drake

Follow this and additional works at: https://researchrepository.wvu.edu/faculty_publications

\section{Digital Commons Citation}

Cassak, P. A. and Drake, J. F., "The impact of microscopic magnetic reconnection on pre-flare energy storage" (2009). Faculty Scholarship. 371.

https://researchrepository.wvu.edu/faculty_publications/371 


\title{
THE IMPACT OF MICROSCOPIC MAGNETIC RECONNECTION ON PRE-FLARE ENERGY STORAGE
}

\author{
P. A. CASSAK ${ }^{1}$ AND J. F. DRake ${ }^{2}$ \\ ${ }^{1}$ Department of Physics, West Virginia University, Morgantown, WV 26506, USA; Paul.Cassak@mail.wvu.edu \\ ${ }^{2}$ Department of Physics and Institute for Research in Electronics and Applied Physics, University of Maryland, College Park, MD 20742, USA; \\ drake@plasma.umd.edu \\ Received 2009 September 19; accepted 2009 November 23; published 2009 December 4
}

\begin{abstract}
It is widely accepted that magnetic reconnection releases a large amount of energy during solar flares. Studies of reconnection usually assume that the length scale over which the global (macroscopic) magnetic field reverses is identical to the thickness of the reconnection site. However, in spatially extended high-Lundquist number plasmas such as the solar corona, this scenario is untenable; the reconnection site is microscopic and embedded inside the macroscopic current set up by global fields. We use numerical simulations and scaling arguments to show that embedded effects on reconnection could have a profound influence on energy storage before a flare. From largescale high-Lundquist number resistive magnetohydrodynamics simulations of reconnection with a diffusion region on a much smaller scale than the macroscopic current sheet, we find that the generation of secondary islands is governed by the local magnetic field immediately upstream of the diffusion region rather than the (potentially much larger) global field. This diminishes the production of secondary islands and leads to a thicker diffusion region than those predicted using the global field strength. Such considerations are crucial for understanding the onset of solar eruptions and how energy accumulates before such eruptions. We argue that if reconnection with secondary islands is fast, the energy storage times before an eruption are too small to explain observations. If reconnection with secondary islands remains slow, embedded effects cause the diffusion region to begin far wider than kinetic scales, so energy storage before a flare can occur while collisional (Sweet-Parker) reconnection with secondary islands proceeds.
\end{abstract}

Key words: Sun: activity - Sun: chromosphere - Sun: corona - Sun: flares - magnetic fields - MHD

\section{INTRODUCTION}

Energy release during solar flares is thought to be allowed by magnetic reconnection. A long-standing question was how reconnection releases energy at the observed rate, which is much faster than the classical Sweet-Parker model (Sweet 1958; Parker 1957, 1963). A leading candidate is collisionless (Hall) reconnection (Birn et al. 2001). An equally important question is how energy accumulates beforehand, a conundrum in light of reconnection. If reconnection is always fast and begins easily, reconnection would release any free energy immediately, precluding major energy storage. Energy accumulation, therefore, requires that reconnection can be slow or completely inactive.

It was recently proposed that Sweet-Parker reconnection, a valid model for some parameters as confirmed by simulations (Biskamp 1986) and experiments (Ji et al. 1998; Trintchouk et al. 2003; Furno et al. 2005), occurs continuously before a flare; its slowness allows energy to accumulate (Cassak et al. 2005, 2006, 2007b, 2008; Uzdensky 2007). The transition from Sweet-Parker to Hall reconnection is catastrophic, implying sudden onset of energy release. Consistent with this scenario, flux emergence (Longcope et al. 2005) and coronal eruption (Liu et al. 2009) observations display slow energy release before fast. Transitions at kinetic scales are observed in experiments (Ren et al. 2005; Egedal et al. 2007). Stellar flare data imply kinetic effects are relevant (Cassak et al. 2008).

This discussion tacitly assumes Sweet-Parker scaling holds for coronal parameters with Lundquist number $S=$ $4 \pi c_{A} L_{\mathrm{SP}} / \eta c^{2} \sim 10^{14}$, where $\eta$ is the resistivity, and $L$ and $c_{A}$ are the characteristic length scale and Alfvén speed. However, this is false. Thin current sheets are unstable to secondary island formation when $S>S_{\text {crit }} \sim 10^{4}$ (Biskamp 1986). Secondary islands, called plasmoids upon ejection from the current layer, are observed in many contexts (Reeves et al. 2008;
Lin et al. 2008; Linton \& Moldwin 2009). No quantitative model of their impact on Sweet-Parker reconnection exists, but evidence suggests it gets faster (Matthaeus \& Lamkin 1986; Lazarian \& Vishniac 1999; Lapenta 2008; Loureiro et al. 2009; Bhattacharjee et al. 2009). Secondary islands make sheets thinner (Shibata \& Tanuma 2001), as confirmed by simulations (Daughton et al. 2009a, 2009b; Cassak et al. 2009). These effects are critical; if secondary islands make reconnection fast or hasten the transition to collisionless reconnection, it is unclear how pre-flare energy accumulates.

In this Letter, we address how energy storage during collisional reconnection is affected by secondary islands, taking into account the large separation between the thicknesses of the equilibrium current layer and the reconnection dissipation layer. Coronal current sheets can be $\gtrsim 10^{3} \mathrm{~km}$ thick (Lin et al. 2009), but the diffusion region is microscopic and embedded deeply within the macroscopic current sheet. This has been studied in magnetospheric contexts (Pritchett \& Coroniti 1995; Sitnov et al. 2000; Yin \& Winske 2002). In embedded systems, the magnetic field immediately upstream of the diffusion region $B_{\text {up }}$ is weaker than the asymptotic (large-scale) field $B_{\text {asymp }}$ (Shay et al. 2004). The diffusion region sees a much smaller effective Lundquist number $S_{\text {up }}$ than the asymptotic value $S_{\text {asymp. The } S}$ in the Biskamp (1986) criterion $S>S_{\text {crit }}$ should be $S_{\text {up }}$ rather than $S_{\text {asymp. }}$. We confirm this prediction using high- $S$ magnetohydrodynamic (MHD) simulations of reconnection embedded in a wide current sheet. Embedded effects suppress secondary island generation and broaden the diffusion region relative to expectations based on $S_{\text {asymp }}$.

To address the impact on flares, we need quantitative models incorporating secondary islands. No accepted theory exists, but recent work by Daughton et al. (2009a) and Loureiro et al. (2007) provides a starting point. We argue that secondary islands are ubiquitous in coronal reconnection. If Sweet-Parker 


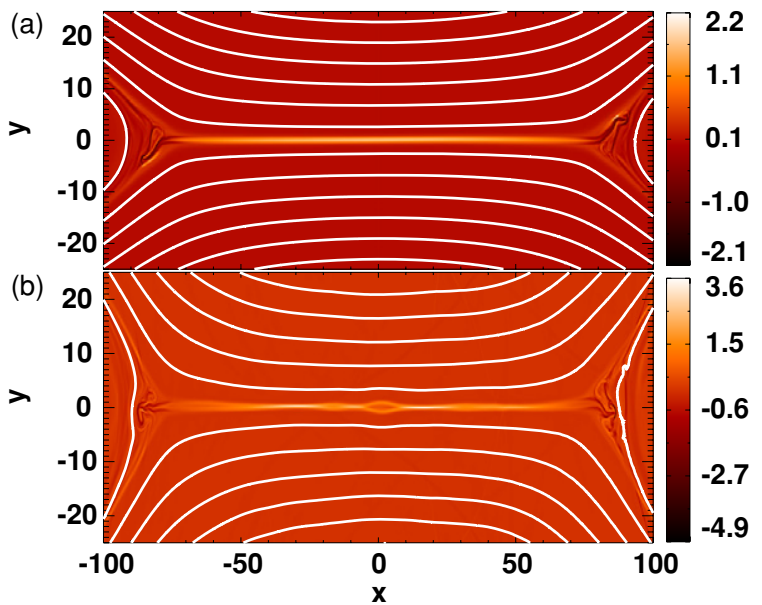

Figure 1. Out-of-plane current density $J_{z}$ for the $w_{0}=10$ simulation with $\eta=0.003$ at (a) $t=4000$ and (b) $t=5500$. Only a small fraction of the domain is plotted and its aspect ratio is altered for clarity.

reconnection with secondary islands is fast, issues of onset and energy storage remain unsolved; if slow, embedded effects ensure sufficient time to store energy before a flare.

\section{SIMULATIONS AND RESULTS}

Two-dimensional resistive MHD simulations are performed using $\mathrm{F}_{3} \mathrm{D}$ (Shay et al. 2004) in a periodic domain of size $L_{x} \times L_{y}=409.6 \times 204.8$ with a grid scale of 0.1 . Lengths, magnetic fields, and densities are normalized to arbitrary values $L_{0}, B_{0}$, and $n_{0}$; velocities, times, electric fields, and resistivities are normalized to the Alfvén speed $c_{A 0}=B_{0} /\left(4 \pi m_{i} n_{0}\right)^{1 / 2}$, the Alfvén time $L_{0} / c_{A 0}, E_{0}=c_{A 0} B_{0} / c$, and $\eta_{0}=4 \pi c_{A 0} L_{0} / c^{2}$, where $m_{i}$ is the ion mass.

The equilibrium is a double Harris sheet $B_{x}(y)=\tanh [(y-$ $\left.\left.L_{y} / 4\right) / W_{0}\right]-\tanh \left[\left(y-3 L_{y} / 4\right) / W_{0}\right]-1$ with current sheet thickness $W_{0}$. The density asymptotes at 1 and is non-uniform to balance total pressure. The temperature $T=1$ is uniform and is not evolved. Resistivity is constant and uniform throughout all simulations. Ohmic heating is ignored. Viscosity is omitted, but fourth-order diffusion with coefficient $3 \times 10^{-5}$ is used in all equations to damp noise at the grid scale. A single $\mathrm{X}$-line is seeded using a coherent magnetic perturbation. Initial random perturbations on the magnetic field of amplitude $5 \times 10^{-6}$ break symmetry so secondary islands are ejected. There is no out-ofplane (guide) magnetic field.

In collisional reconnection, $L \sim L_{\mathrm{SP}}$, the half-length of the Sweet-Parker diffusion region. In periodic systems, $L_{\mathrm{SP}} \sim$ $L_{x} / 4 \sim 102.4$. We find $L_{\mathrm{SP}}$ closer to 75 . To test the Biskamp (1986) criterion, we use a base simulation with $\eta=0.003$, corresponding to $S_{\text {asymp }} \simeq 2.5 \times 10^{4}$, which is unstable to secondary islands when $B_{\text {up }} \simeq B_{\text {asymp. The classical Sweet- }}$ Parker thickness is $\delta_{\mathrm{SP}} \simeq 0.47$, so reconnection is initially embedded if $W_{0}>0.47$.

As a first test, we run a simulation with $W_{0}=0.4$. The diffusion region is immediately unstable to secondary tearing as expected, splintering into $\sim 15$ pieces at early times. Next, we use $W_{0}=5,10$, and 15 . For these runs, $S_{\text {asymp }}>S_{\text {crit }}$, but $S_{\text {up }}<S_{\text {crit }}$ because $B_{\text {up }}$ is substantially decreased. The simulations reveal that secondary islands do not form initially, suggesting that $S_{\text {up }}$ is the control parameter. Figure 1 shows the out-of-plane current density $J_{z}$ for the $W_{0}=10$ simulation at (a) $t=4000$ and (b) $t=5500$, revealing secondary island formation only later in the evolution.

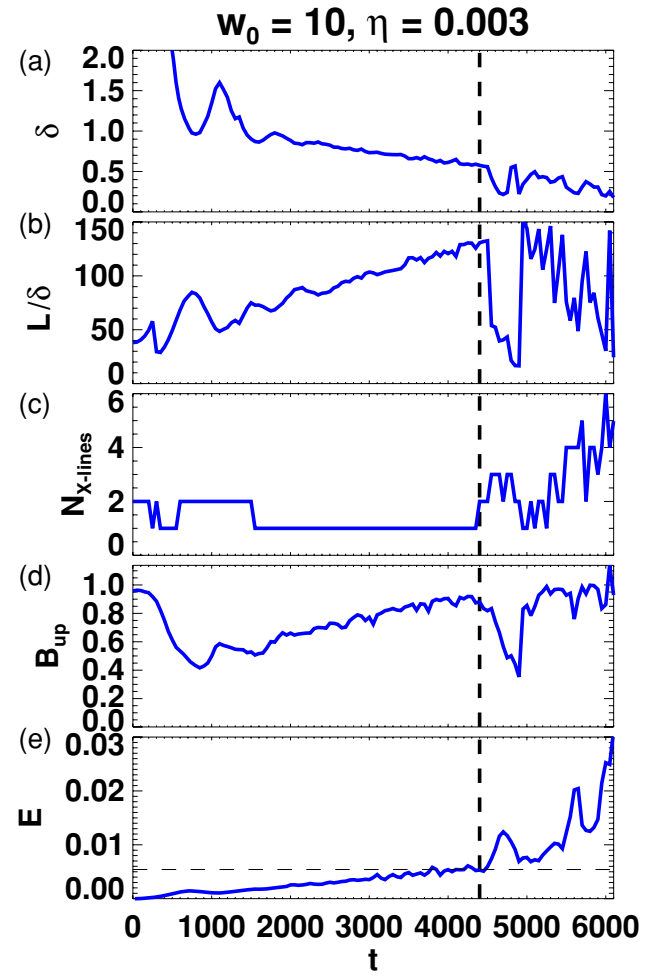

Figure 2. Quantities as a function of time $t$ for the $w_{0}=10, \eta=0.003$ simulation. (a) Diffusion region (half) thickness $\delta$, (b) aspect ratio of the diffusion region $L / \delta$, (c) number of X-lines, (d) magnetic field strength upstream of the main X-line, and (e) reconnection electric field. The vertical dashed line denotes the time at which secondary islands appear.

In embedded reconnection, the initial half-thickness $\delta_{i}$ is much larger than $\delta_{\mathrm{SP}}$ based on $B_{\text {asymp }}$. To estimate $\delta_{i}$, classical Sweet-Parker theory predicts (Parker 1957)

$$
\frac{\delta_{i}}{L_{\mathrm{SP}}} \sim S_{\mathrm{up}}^{-1 / 2} \sim \sqrt{\frac{\eta c^{2}}{4 \pi c_{A, \mathrm{up}} L_{\mathrm{SP}}}},
$$

where $c_{A \text {,up }}$ is the Alfvén speed based on $B_{\text {up }}$. If the diffusion region is deeply embedded $\left(W_{0} \gg \delta_{i}\right)$, the field profile is roughly linear, $B_{\text {up }} \simeq B_{\text {asymp }} \delta_{i} / W_{0}$. Eliminating $B_{\text {up }}$ gives

$$
\frac{\delta_{i}}{L_{\mathrm{SP}}} \sim S_{\text {asymp }}^{-1 / 3}\left(\frac{W_{0}}{L_{\mathrm{SP}}}\right)^{1 / 3},
$$

so $\delta_{i} \gg \delta_{\mathrm{SP}}$ based on $B_{\text {asymp }}$ for large $S$.

The dynamical evolution is completely self-generated. In embedded reconnection, the field upstream of the diffusion region is stronger than $B_{\text {up }}$. This stronger field is frozen-in to the reconnection inflow. Therefore, the reconnection inflow itself carries in the stronger field. From Equation (1), increasing $B_{\text {up }}$ causes thinner diffusion regions. When $B_{\text {up }}$ is large enough to cross the Biskamp criterion, secondary island production begins.

This dynamics can be seen in Figure 2. Panel (a) shows the diffusion region thickness $\delta$, measured as the $e$-folding distance of $J_{z}$ in the inflow direction across the main X-line, as a function of time for the $W_{0}=10$ simulation, which is representative. Following transients which last until $t \sim 2300, \delta$ decreases steadily in time. Extrapolating the linear part in back in time to $t=0$, we find $\delta_{i} \simeq 1.2$, in good agreement with $\delta_{i} \simeq 1.3$ predicted from Equation (2).

Since the diffusion region half-length $L$, measured as the $e$-folding distance of $J_{z}$ in the outflow direction, is relatively 


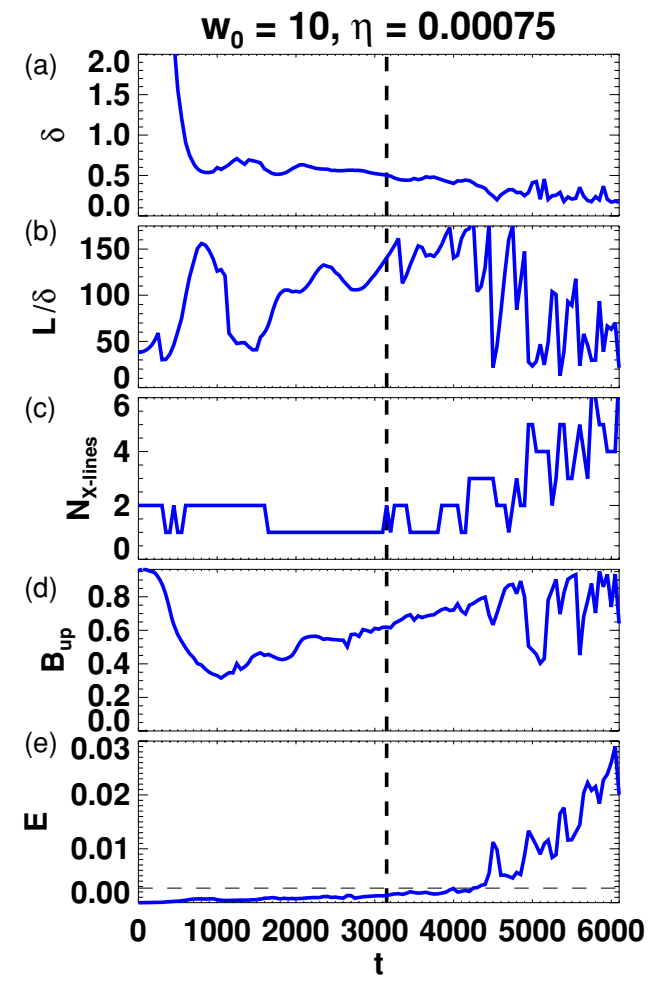

Figure 3. Same quantities as plotted in Figure 2 for the simulation with $w_{0}=10$ but with a smaller resistivity of $\eta=0.00075$.

constant, the aspect ratio $L / \delta$ steadily increases (panel (b)). Only when $L / \delta \simeq 130$ does secondary island formation ensue. This is shown in (c), with the number $N$ of $\mathrm{X}$-lines as a function of time. To find $N, \mathrm{X}$-line candidates are identified as locations where the reconnected field $B_{y}$ changes sign along the neutral line. The number of active $\mathrm{X}$-lines is hand-counted to determine $N$. After initial transients, the system has a single $X$-line for $\simeq 2000$ time units. When $L / \delta$ exceeds $130, N$ increases. Panel (d) shows $B_{\text {up }}$, measured as the reconnecting field strength $2 \delta$ upstream of the main X-line, which steadily increases as reconnection proceeds. The onset of secondary islands at $L / \delta \simeq 130$ (corresponding to $S_{\text {up }} \sim 1.7 \times 10^{4}$ ) agrees with the Biskamp (1986) criterion.

To confirm $B_{\text {up }}$ is the relevant field, we perform a simulation with $\eta$ reduced by a factor of 4 (corresponding to $S_{\text {asymp }} \sim 10^{5}$ ), which should require a smaller $B_{\text {up }}$ to induce secondary tearing. Figure 3 shows plots like Figure 2 . As before, transients last until $t \simeq 2000$, then the sheet thins as $B_{\text {up }}$ increases. The extrapolated $\delta_{i} \simeq 0.8$ agrees well with the prediction of $\delta_{i} \simeq 0.8$ from Equation (2). There is only a single X-line until $t \simeq 3200$, when secondary islands form. At this time, $L / \delta \simeq 130$ as in the previous simulation. However, $B_{\text {up }} \sim 0.6$, rather than 0.9 , consistent with expectations. We conclude that the field that controls the onset of secondary islands is $B_{\text {up }}$, not $B_{\text {asymp }}$.

The reconnection electric field $E$ is plotted in panel (e) of Figures 2 and 3, with the classical Sweet-Parker prediction $E_{\mathrm{SP}}$ based on $B_{\text {asymp plotted as the dashed line. Clearly, } E \text { exceeds }}$ $E_{\mathrm{SP}}$ upon onset of secondary islands. However, we cannot ascertain whether $E$ reaches a steady value. Larger simulations are required to determine whether $E$ is slower than or comparable to the fast reconnection rate of $E \sim 0.1$.

It is crucial to ensure that secondary islands do not introduce excessive grid scale dissipation, which could adversely affect the results. Cuts of $J_{z}$ in the inflow direction for the data shown in Figure 1 are shown in Figure 4(a) before and (b) after secondary

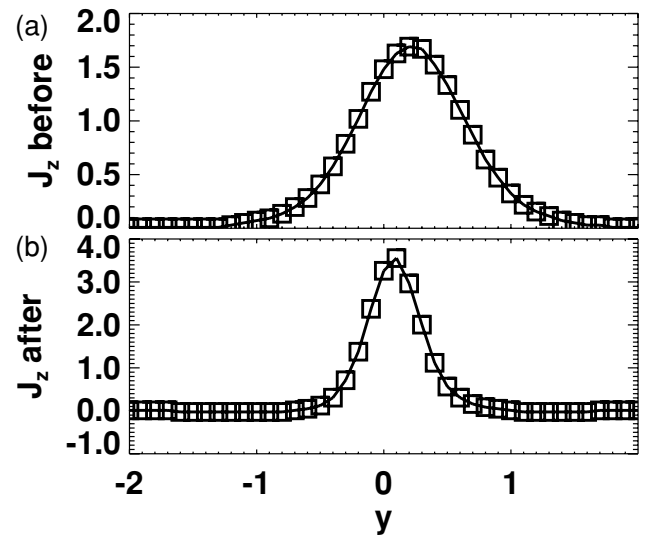

Figure 4. Cut across $J_{z}$ in the inflow direction of the two current sheets pictured in Figure 1 confirming sufficient numerical resolution.

islands arise. There are over 16 and 10 grid cells across the diffusion region, respectively, confirming that grid scale effects are negligible.

\section{APPLICATIONS TO THE CORONA}

The present work has important implications for pre-flare energy storage in the solar corona. The most important issues are whether secondary islands (1) make Sweet-Parker reconnection fast, (2) hasten the transition to collisionless reconnection, and (3) make the reconnection electric field exceed the Dreicer field, which would invalidate the use of a Spitzer resistivity. We address each in turn.

At present, no accepted quantitative model for the impact of secondary islands exists. Daughton et al. (2009a) provide a possible starting point by assuming that fragments of the diffusion region obey the Sweet-Parker model, allowing one to predict the scaling with the number of islands $N$. With $N$ islands, the length $L$ of each segment scales like $L \sim L_{\mathrm{SP}} / N$. From Equation (1), the thickness of the fragmented layer scales like

$$
\delta \sim \frac{\delta_{\mathrm{SP}}}{N^{1 / 2}},
$$

where $\delta_{\mathrm{SP}}$ is the classical Sweet-Parker prediction. The layer becomes thinner, as noted previously (Shibata \& Tanuma 2001). Since $E \sim \delta / L$,

$$
E \sim E_{\mathrm{SP}} N^{1 / 2}
$$

where $E_{\mathrm{SP}}$ is the classical Sweet-Parker prediction. As previously noted, $E$ increases due to secondary islands. Evidence that this scaling holds in a time averaged sense is presented in a companion study (Cassak et al. 2009).

There is evidence that the number of islands $N$ scales with a power of $S$. The analytical model by Loureiro et al. (2007) predicted $N \propto S^{3 / 8}$. Amending their result to include embedded effects, we write

$$
N \sim\left(\frac{S_{\text {up }}}{S_{\text {crit }}}\right)^{\alpha},
$$

where $S_{\text {crit }} \sim 10^{4}$ and $\alpha$ generalizes the model by characterizing potential models of secondary island generation. Recent numerical results by Samtaney et al. (2009) agree with the prediction, while Daughton et al. (2009a) report $\alpha \simeq 0.6$ in particle-in-cell simulations, and Cassak et al. (2009) report $\alpha \sim 0.72-0.93$ in MHD simulations. 
While the appropriateness of these models for coronal parameters has not been shown, it is instructive to investigate the predictions to see the effect of embedding and secondary islands. We consider properties of the reconnection for various $\alpha$. To compare with coronal data, we use representative pre-flare parameters of $B_{\text {asymp }} \sim 100 \mathrm{G}, n \sim 3 \times 10^{9} \mathrm{~cm}^{-3}, L_{\mathrm{SP}} \sim 10^{9} \mathrm{~cm}$, and $T \sim 10^{6} \mathrm{~K}$ (Cassak et al. 2006). Using a parallel Spitzer resistivity, this gives $S_{\text {asymp }} \sim 4 \times 10^{13}$. For the macroscopic current sheet thickness, we use $W_{s} \sim 10^{3} \mathrm{~km}$ (Lin et al. 2009). These values do not apply to a particular event; the focus is on orders of magnitude, not specific values of predictions.

The reconnection rate. Using $E_{\mathrm{SP}} \sim S_{\mathrm{up}}^{-1 / 2}$ with Equations (4) and (5) implies $E \propto S_{\text {up }}^{-(1-\alpha) / 2}$, which is quite sensitive to $\alpha$. If $\alpha \simeq 1$, then $E$ weakly depends on $S$ and reconnection is fast. If $\alpha<1$, then $E$ scales with a negative power of $S$, so reconnection is slow. To make this quantitative, ignore embedded effects for simplicity (which make reconnection slower). The classical (normalized) prediction is $E_{\mathrm{SP}} \sim S_{\text {asymp }}^{-1 / 2} \sim 10^{-7}$, six orders of magnitude slower than observed energy release rates (Parker 1963). To put this in perspective, if $\alpha=3 / 8$, then $E \propto S_{\text {up }}^{-5 / 16}$ giving $E \sim 63 E_{\mathrm{SP}}$ for coronal parameters, which is four orders of magnitude slower than observed rates; see Cassak et al. (2009) for further discussion.

The transition to collisionless reconnection. Collisionless reconnection begins when $\delta$ reaches a kinetic length scale (Aydemir 1991; Mandt et al. 1994; Ma \& Bhattacharjee 1996), which we call $\delta_{k}$. The transition is abrupt; for anti-parallel reconnection, $\delta_{k}=d_{i}=c / \omega_{p i}$, the ion inertial scale (Cassak et al. 2005), where $\omega_{p i}$ is the ion plasma frequency. With a strong guide field $\delta_{k}=\rho_{s}=c_{s} / \Omega_{c i}$, the ion Larmor radius (Cassak et al. 2007a), where $c_{s}$ is the ion sound speed and $\Omega_{c i}$ is the ion cyclotron frequency. Reconnection in the corona has a strong guide field (Uzdensky 2007), so the $\delta_{k}=\rho_{s}$ is appropriate.

If reconnection with secondary islands remains slow $(\alpha<1)$, one must ask how secondary islands impact onset of collisionless reconnection. We estimate the length scales for the corona. The kinetic scale is $\rho_{s} \sim 10 \mathrm{~cm}$. From Equation (2), the initial thickness of the diffusion region is $\delta_{\mathrm{SP}, \mathrm{i}} \sim\left(W_{s} L_{\mathrm{SP}}^{2} / S_{\text {asymp }}\right)^{1 / 3} \simeq$ $10^{4} \mathrm{~cm}$. The initial upstream field is $B_{\mathrm{up}, \mathrm{i}} \sim B_{\mathrm{asymp}} \delta_{\mathrm{SP}, \mathrm{i}} / W_{s} \simeq$ $0.01 \mathrm{G}$, giving an initial $S_{\text {up }, \mathrm{i}} \sim 5 \times 10^{9}$. Since $S_{\text {up }, \mathrm{i}} \gg S_{\text {crit }} \sim$ $10^{4}$, secondary islands occur during coronal Sweet-Parker reconnection from the outset despite embedded effects. For definiteness, if $\alpha=3 / 8$, Equation (5) predicts initially $N_{i} \sim 140$ islands. From Equation (3), $\delta_{i} \sim \delta_{\mathrm{SP}, \mathrm{i}} / N_{i}^{1 / 2} \sim 1200 \mathrm{~cm}$. This is $\sim 10^{2}$ times wider than $\rho_{s}$, so the fragmented diffusion regions are initially wider than kinetic scales. Reconnection at initiation is collisional.

To estimate the critical upstream field $B_{\text {up,c }}$ needed to make $\delta \sim \delta_{k}$, set Equation (3) equal to $\delta_{k}$ and eliminate $N$ using Equation (5). We make contact with previous work by first writing this in terms of a dimensionless effective collisionality parameter $\eta_{c}=\eta c^{2} / 4 \pi c_{A \text {,up }} \delta_{k}$, getting

$$
\eta_{c}=\frac{1}{S_{\mathrm{crit}}^{\alpha /(1+\alpha)}}\left(\frac{\delta_{k}}{L_{\mathrm{SP}}}\right)^{(1-\alpha) /(1+\alpha)}
$$

This generalizes the result by Daughton et al. (2009a) to include embedded effects ( $B_{\text {up }, \mathrm{c}}$ instead of $B_{\text {asymp }}$ in $\eta_{c}$ ), guide field effects $\left(\delta_{k}\right.$ instead of $\left.d_{i}\right)$, and the amended Loureiro et al. (2007)

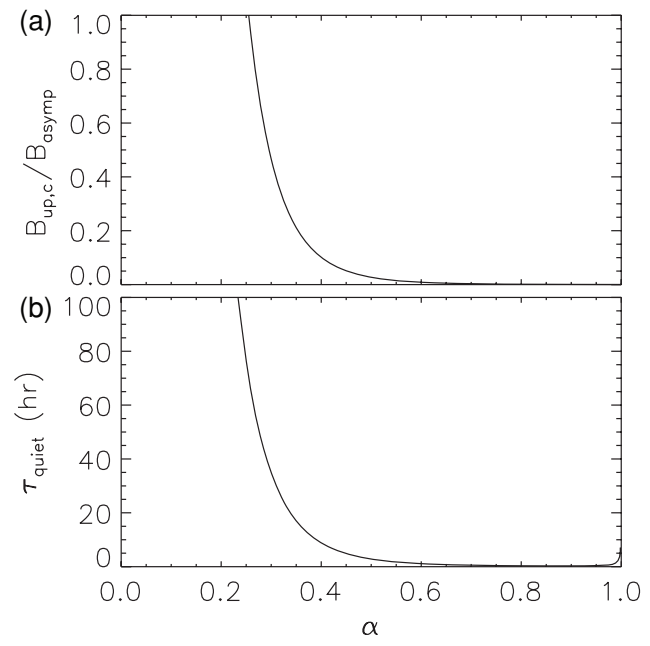

Figure 5. From the model, the predicted critical field $B_{\text {up,c }}$ and quiet time $\tau_{\text {quiet }}$ as a function of the exponent $\alpha$ in the secondary island generation model for coronal parameters employed in the text.

result in Equation (5). Solving for $B_{\text {up,c }}$ gives

$$
B_{\text {up }, \mathrm{c}} \sim B_{\text {asymp }} \frac{S_{\text {crit }}^{\alpha /(1+\alpha)}}{S_{\text {asymp }}}\left(\frac{L_{\mathrm{SP}}}{\delta_{k}}\right)^{2 /(1+\alpha)} .
$$

This result is plotted as a function of $\alpha$ in Figure 5(a) using $\delta_{k}=\rho_{s}$. For very small $\alpha, B_{\text {up }, \mathrm{c}}>B_{\text {asymp }}$, so a flare would never occur $(\alpha \lesssim 0.25)$. For larger $\alpha, B_{\text {up }, \mathrm{c}} \ll B_{\text {asymp }}$, so a flare would onset too easily to store energy $(\alpha \gtrsim 0.5)$.

The time it takes for this upstream field to convect into the diffusion region is a measure of the quiet time before a flare $\tau_{\text {quiet }}$. We estimate it using an analysis similar to Shay et al. (2004); Cassak et al. (2006). The time it takes to convect flow is $\tau_{\text {quiet }} \sim \int_{\delta_{i}}^{\delta_{f}} d \delta / v_{\text {in }}$, where the bounds of integration are the initial and final thicknesses. We write $v_{\text {in }}$ in terms of $\delta$ using conservation of mass $v_{\text {in }} \sim c_{A \text {,up }} \delta / L$. Then Equation (4) implies $\delta / L \sim \delta_{\mathrm{SP}} N^{1 / 2} / L_{\mathrm{SP}}$. Finally, using Equation (5) to eliminate $N$ and $B_{\text {up }} \sim B_{\text {asymp }} \delta / W_{s}$ gives the integrand entirely in terms of $\delta$ and asymptotic system parameters. Integrating from the distance upstream that the field strength equals the critical field $\delta_{i} \sim \delta_{\text {up }, \mathrm{c}} \sim\left(B_{\text {up }, \mathrm{c}} / B_{\text {asymp }}\right) W_{s}$ to very small kinetic scales $\delta_{f} \sim 0$ gives a quiet time

$$
\tau_{\text {quiet }} \simeq \frac{2}{1-\alpha} \frac{W_{s}}{c_{A, \text { asymp }}} S_{\text {crit }}^{\alpha /(1+\alpha)}\left(\frac{L_{\mathrm{SP}}}{\delta_{k}}\right)^{(1-\alpha) /(1+\alpha)} .
$$

This is plotted (in hours) in Figure 5(b) as a function of $\alpha$. For $0.25 \lesssim \alpha \lesssim 0.5$, there are several hours for energy storage, which is a reasonable pre-flare storage time (Priest \& Forbes 2002). Since $\tau_{\text {quiet }}$ is linear in $W_{s}$, wider macroscopic sheets produce longer quiet times. We conclude that secondary islands do not invalidate recent models (Cassak et al. 2005, 2006; Uzdensky 2007; Cassak et al. 2008) of energy storage provided $\alpha$ is in this range (for the parameters and secondary island model employed here). If $\alpha \simeq 1$, the quiet time before a flare would be exceedingly small, so energy would not have time to accumulate.

The Dreicer field. The Dreicer field is

$$
E_{D} \sim 0.43\left(\frac{2 \pi n_{e} Z e^{3}}{k_{B} T_{e}} \ln \Lambda\right) \sim 1.25 \times 10^{-7} \text { statvolt } \mathrm{cm}^{-1} .
$$


Using $\alpha=3 / 8$ and the asymptotic fields, $E \sim 1.3 \times 10^{-5}$ statvolt $\mathrm{cm}^{-1}$, which exceeds the Dreicer field. However, from Figure 5(a), the transition to collisionless reconnection occurs when $B_{\text {up }} \sim 10 \mathrm{G}$. For these fields, $E \sim 2.6 \times 10^{-7}$ statvolts $\mathrm{cm}^{-1}$, comparable to the Dreicer field. In this scenario, the field during the quiet time is below the Dreicer field, so the description using a Spitzer resistivity is valid.

\section{DISCUSSION}

The precise scaling of reconnection with secondary islands remains an open question. The model employed here, for example, does not take into account possible hierarchical secondary island scenarios (Shibata \& Tanuma 2001; Daughton et al. 2009a; Bhattacharjee et al. 2009). Regardless, the present results conclusively show that embedded effects can be considerable, and need to be taken into account when modeling coronal reconnection.

In addition to coronal reconnection, the present results may be relevant to chromospheric reconnection (Litvinenko 1999; Chae et al. 2003; Litvinenko \& Chae 2009), where $S \sim 10^{6}-10^{8}$. The inferred reconnection rate exceeds the classical Sweet-Parker prediction by a factor of a few. Using Equation (4) with $\alpha=3 / 8$ predicts an enhancement by a factor of about 5 for $S_{\text {asymp }} \sim 10^{8}$, which brings the observations into closer agreement with theory.

Limitations to the present simulations include the absence of a guide field, the usage of a constant resistivity instead of a Spitzer resistivity, and the omission of Ohmic heating and viscosity; the latter of which may be important for secondary island generation (Park et al. 1984). Three-dimensional effects are omitted, but are potentially important. The models employed here treat only self-generated secondary islands, not externally driven turbulence (Lazarian \& Vishniac 1999; Smith et al. 2004; Kowal et al. 2009; Loureiro et al. 2009).

The authors thank W. Daughton, A. A. Schekochihin, and M. A. Shay for valuable discussions. Computations used resources at the National Energy Research Scientific Computing Center. P.A.C. and J.F.D. acknowledge support from NSF grants PHY-0902479 and PHY-0316197, respectively.

\section{REFERENCES}

Aydemir, A. Y. 1991, Phys. Fluids B, 3, 3025

Bhattacharjee, A., Huang, Y.-M., Yang, H., \& Rogers, B. N. 2009, Phys. Plasmas, 16, 112102

Birn, J., et al. 2001, J. Geophys. Res., 106, 3715

Biskamp, D. 1986, Phys. Fluids, 29, 1520

Cassak, P. A., Drake, J. F., \& Shay, M. A. 2006, ApJ, 644, L145
Cassak, P. A., Drake, J. F., \& Shay, M. A. 2007a, Phys. Plasmas, 14, 054502

Cassak, P. A., Drake, J. F., Shay, M. A., \& Eckhardt, B. 2007b, Phys. Rev. Lett., 98, 215001

Cassak, P. A., Mullan, D. J., \& Shay, M. A. 2008, ApJ, 676, L69

Cassak, P. A., Shay, M. A., \& Drake, J. F. 2005, Phys. Rev. Lett., 95, 235002

Cassak, P. A., Shay, M. A., \& Drake, J. F. 2009, Phys. Plasmas, in press

Chae, J., Moon, Y.-J., \& Park, S.-Y. 2003, J. Korean Astron. Soc., 36, S13

Daughton, W., Roytershteyn, V., Albright, B. J., Karimabadi, H., Yin, L., \& Bowers, K. J. 2009a, Phys. Rev. Lett., 103, 065004

Daughton, W., Roytershteyn, V., Albright, B. J., Karimabadi, H., Yin, L., \& Bowers, K. J. 2009b, Phys. Plasmas, 16, 072117

Egedal, J., Fox, W., Katz, N., Porkolab, M., Reim, K., \& Zhang, E. 2007, Phys. Rev. Lett., 98, 015003

Furno, I., Intrator, T. P., Hemsing, E. W., Hsu, S. C., Abbate, S., Ricci, P., \& Lapenta, G. 2005, Phys. Plasmas, 12, 055702

Ji, H., Yamada, M., Hsu, S., \& Kulsrud, R. 1998, Phys. Rev. Lett., 80, 3256

Kowal, G., Lazarian, A., Vishniac, E. T., \& Otmianowska-Mazur, K. 2009, ApJ 700,63

Lapenta, G. 2008, Phys. Rev. Lett., 100, 235001

Lazarian, A., \& Vishniac, E. 1999, ApJ, 517, 700

Lin, J., Cranmer, S. R., \& Farrugia, C. J. 2008, J. Geophys. Res., 113, A11107

Lin, J., Li, J., Ko, Y.-K., \& Raymond, J. C. 2009, ApJ, 693, 1666

Linton, M. G., \& Moldwin, M. B. 2009, J. Geophys. Res., 114, A07213

Litvinenko, Y. E. 1999, ApJ, 515, 435

Litvinenko, Y. E., \& Chae, J. 2009, Astron. Astrophys., 495, 953

Liu, R., Wang, H. M., \& Alexander, D. 2009, ApJ, 696, 121

Longcope, D. W., McKenzie, D. E., Cirtain, J., \& Scott, J. 2005, ApJ, 630, 596

Loureiro, N. F., Schekochihin, A. A., \& Cowley, S. C. 2007, Phys. Plasmas, 14, 100703

Loureiro, N. F., Uzdensky, D. A., Schekochihin, A. A., Cowley, S. C., \& Yousef, T. A. 2009, MNRAS, 399, L146

Ma, Z. W., \& Bhattacharjee, A. 1996, Geophys. Res. Lett., 23, 1673

Mandt, M. E., Denton, R. E., \& Drake, J. F. 1994, Geophys. Res. Lett., 21, 73

Matthaeus, W. H., \& Lamkin, S. L. 1986, Phys. Fluids, 29, 2513

Park, W., Monticello, D. A., \& White, R. B. 1984, Phys. Fluids, 27, 137

Parker, E. N. 1957, J. Geophys. Res., 62, 509

Parker, E. N. 1963, ApJ, 8, 177

Priest, E. R., \& Forbes, T. R. 2002, Astron. Astrophys. Rev., 10, 313

Pritchett, P. L., \& Coroniti, F. V. 1995, J. Geophys. Res., 100, 23551

Reeves, K. K., et al. 2008, J. Geophys. Res., 113, A00B02

Ren, Y., Yamada, M., Gerhardt, S., Ji, H., Kulsrud, R., \& Kuritsyn, A. 2005, Phys. Rev. Lett., 95, 005003

Samtaney, R., Loureiro, N. F., Uzdensky, D. A., Schekochihin, A. A., \& Cowley, S. C. 2009, Phys. Rev. Lett., 103, 105004

Shay, M. A., Drake, J. F., Swisdak, M., \& Rogers, B. N. 2004, Phys. Plasmas, 11,2199

Shibata, K., \& Tanuma, S. 2001, Earth Planets Space, 53, 473

Sitnov, M. I., Zelenyi, L. M., Malova, H. V., \& Slarma, A. S. 2000, J. Geophys. Res., 105, 13029

Smith, D., Ghosh, S., Dmitruk, P., \& Matthaeus, W. H. 2004, Geophys. Res. Lett., 31, L02805

Sweet, P. A. 1958, in Electromagnetic Phenomena in Cosmical Physics, ed. B. Lehnert (New York: Cambridge Univ. Press), 123

Trintchouk, F., Yamada, M., Ji, H., Kulsrud, R. M., \& Carter, T. A. 2003, Phys. Plasmas, 10, 319

Uzdensky, D. A. 2007, ApJ, 671, 2139

Yin, L., \& Winske, D. 2002, J. Geophys. Res., 107, 1485 